\title{
Exploring a new method based on generalized eigenvalues for the trust-region subproblem
}

\section{Jean Carlos A. Medeiros*, Sandra Augusta Santos.}

\section{Abstract}

In 2017, a method for solving the trust-region subproblem using generalized eigenvalues was rediscovered and improved. In 1989, when the original method was proposed, it presented a poor performance, which was caused by the low quality of eigensolvers available at that time. In this work we explore some geometric characteristics of this method.

\section{Key words:}

unconstrained minimization, eigenvalue problem, trust-region subproblem.

\section{Introduction}

The trust-region methods are iterative methods for numerically solving minimization problems, not only unconstrained but also constrained ones. They consist of defining a quadratic model for the objective function $f$ from a current point $x^{k}$ and establishing a closed ball centered on $x^{k}$ and with radius $\Delta$; this neighborhood around $x^{k}$ is called trust region, because in this region we will trust that the model generates a good approximation for the objective function; then each iteration will have a subproblem of minimizing the model subject to the trust region, thereby generating a sequence of approximations to the solution of the problem, ie the objective function minimizer. Recently, Adachi et al. ${ }^{1}$, based on Gander et al. ${ }^{2}$, developed a method adressing the subproblem in a non-iterative way, solving only one generalized eigenvalue problem. This work investigates the usage of this strategy for solving low dimensional unconstrained minimization problems. The visual appeal provides an additional tool for exploring the geometric features of this approach.

\section{Results and Discussion}

The method is based on using a pencil matrix $A+\lambda B$, where $A$ and $B$ are square matrices of order $n$, and $\lambda>0$ is a real scalar, to construct another pencil matrix of order $2 n+1$, define as

$$
M(\lambda)=\left(\begin{array}{ccc}
\Delta^{2} & 0 & g^{T} \\
0 & -B & A+\lambda B \\
g & A+\lambda B & O_{n}
\end{array}\right)
$$

or a pencil matrix of order $2 \mathrm{n}$, if we want to work with a dense pencil instead of a sparse one, which we define as

$$
\widetilde{M}(\lambda)=\left(\begin{array}{cc}
-B & A+\lambda B \\
A+\lambda B & \frac{-g g^{T}}{\Delta^{2}}
\end{array}\right),
$$

in which $g=\nabla f\left(x^{k}\right)$ and $A \approx \nabla^{2} f\left(x^{k}\right)$. The solution of the subproblem will be the eigenvector associated with the largest finite generalized eigenvalue of the pencils. Another difference between this method and the traditional ones is in the choice of the norm used to restrict the solution to the trust region. In general the Euclidean norm is used, so all directions from the center have the same Euclidean norm. However, it is very common that the scales of the variables have a different order of magnitude (see section 6.7.1 from Conn et al. ${ }^{3}$ ), then the method will prioritize the directions of greater deformation of the model using the energy norm. Therefore at each iteration we will define the hessian $A$ of the model as being the hessian of $f$, and since $f$ is of class $C^{2}, A$ admits spectral decomposition $A=V D V^{\top}$, so we define $B=V|D| V^{\top}$. In this way we guarantee that $B$ has the same preferential directions of the Hessian of the objective function at the current point, and also ensure that it is positive definite, thus defining the energy norm using $\mathrm{B}$.

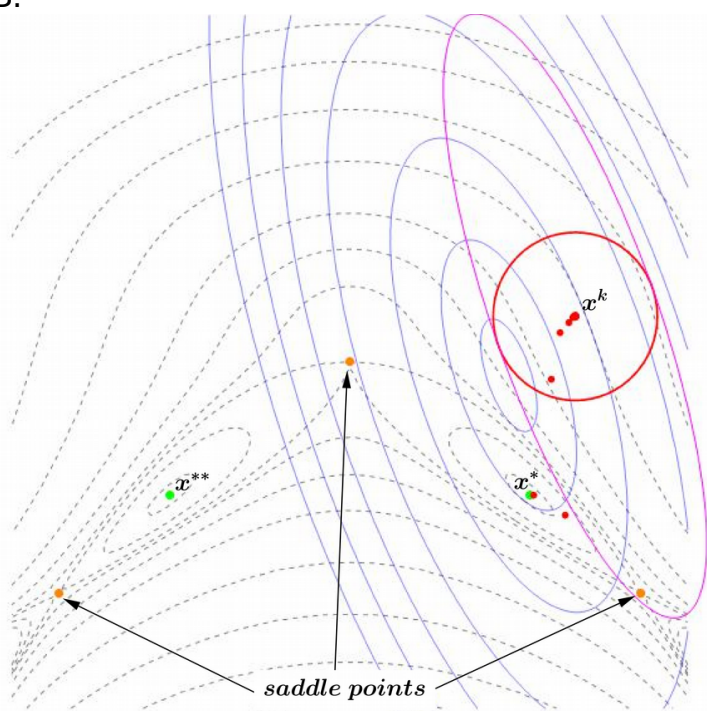

Image 1. Comparison between the region constructed with the Euclidean norm (red) and energy norm (blue).

\section{Conclusions}

Briefly, by allowing to work with a matrix $B \neq I_{n}$, the trust region fits in to the geometry of the model at each iteration and even though the construction of matrix $B$ may offer some limitations, the method is very efficient.

\section{Acknowledgement}

We thank CNPq for the financial support and the authors Adachi et al. ${ }^{1}$, who left available online their Matlab code.

${ }^{1}$ Adachi, S.; Iwata, S.; Nakatsukasa, Y.; Takeda, A. Solving the Trust-Region Subproblem By a Generalized Eigenvalue Problem. SIAM Journal on Optimization, 27(1):269-291, 2017. doi: https://doi.org/10.1137/16M1058200.

${ }^{2}$ Gander, W.; Golub, G.H.; von Matt, U. A Constrained Eigenvalue Problem. Linear Algebra and its Applications, 114:815-839, $1989 . \quad$ doi: https://doi.org/10.1016/0024-3795(89)90494-1.

${ }^{3}$ Conn, A. R.; Gould, N. I. M.; Toint, P. L. Trust-region methods. SIAM, Philadelphia, 2000. URL https://doi.org/10.1137/1.9780898719857. 